\title{
An Advanced Approach for Barcode Modulation Using High Capacity 2D Barcode Technique
}

\author{
Vidyashree Kokane ${ }^{1}$, Shyam Gupta ${ }^{2}$ \\ ${ }^{1}$ PG Student, Siddhant College of Engineering, Sudumbre, Savitribai Phule Pune University \\ ${ }^{2}$ Professor, Computer Department, Siddhant College of Engineering, Sudumbre, Savitribai Phule Pune University
}

\begin{abstract}
Barcoding techniques have boosted a lot in these recent days by making innovative techniques of barcoding larger data into smaller spaces and encode the data into a small barcode for making it readable over handheld devices such as barcode scanners and scanning machines. Using the traditional barcoding techniques requires a predefined barcode fonts and barcode scanning hardwares, which is the major drawback of the traditional system. The proposed system designs a novel barcode generation and barcoding reading technique without a need of hardware scanner and no additional hardware for interface between barcode generating and reading devices. The barcode generation of higher capacity is proposed using a new hashing technique, not used previously before in barcoding systems, for reducing the space required for storing the data in barcodes. In this paper, HC2D barcode i.e. High capacity $2 D$ barcode technique for large capacity of barcodes is proposed considering the current market trend of android devices and need of large data encoding and secure transmission of data with less transmission overheads. Thus the proposed system designs novel HC2D barcode generation and reading technique along with secure and efficient barcode transmission technique to ensure the data security.
\end{abstract}

Keywords: HC2D barcode, data transfer, Barcode encoding, barcode decoding, Android platform

\section{Introduction}

Now a days most of the identification systems make efficient use of barcodes since its invention i.e. 1952 [1]. Rather the commercial products being sold in market are signed with the barcodes to determine the cost of the product with the use of hardware scanners. Faster and even more reliable barcoding techniques are designed for storing the digital data of products. Considering these cost-effective barcodes as well as their wider use in recent times opened a new fore front by generation of HC2D barcodes in scenarios like larger data storage which requires complex architectures, have gained lot of interest with use of QR codes [2]. Performance of the various barcoding techniques such as 1D barcode, 2D barcode, PDF457 and QR Codes using camera applications has emerged as a new trend for data digitalization and data storage with limited space resources. [3].

With the use of a series of HC2D barcode transmission of data over two or more digital mobile phones is proposed, compatible to mobiles phones making it feasible for using it over speed of $10 \mathrm{kpbs}$ bit rate. With higher bit rates of about $14 \mathrm{Mbps}$ is done by making use of a digital camera and computer system along with decoding logic for the barcode. For transferring data, traditional systems make use of physical transmission medium, such as printing over books or printing over products. Recently the barcode transmission were done using QPSK Quadrature Phase Shift Keying systems.

\section{Literature Survey}

A. QR Code Detection in Arbitrarily Acquired Images Through creation of links between Internet resources and physical objects, there enable rich context interaction
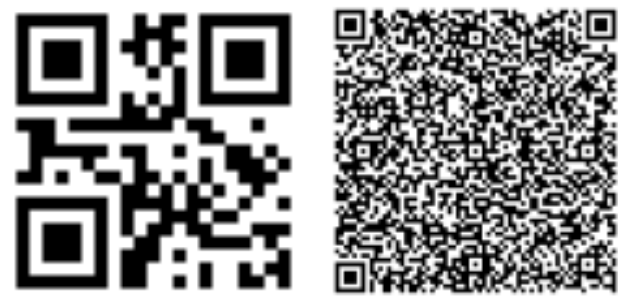

Figure 1: Different versions of QR code

applications of Quick Response (QR) codes. There are this kind of barcode applications are not common in spite of the widespread use for people and robots which are visually impaired because during image acquisition that the symbol is properly by existing decoders framed are assumed. To perform accurate detection of $\mathrm{QR}$ code symbols a two-stage component-based approach proposed by this work in arbitrarily acquired images.

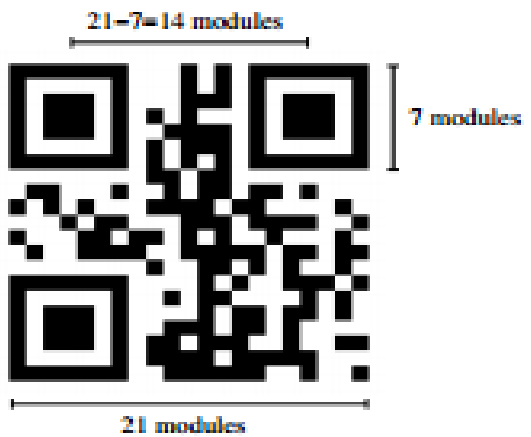

Figure 2: 2D QR code

With the help of the framework which detect the objects proposed by Viola-Jones to detect parts of the symbol is trained, a cascade classifier in the first stage. In the second stage, in order to evaluate the detected patterns are aggregated if they are spatially arranged with the components of a QR code symbol in a way that is geometrically consistent. There was performed an extensive 


\section{International Journal of Science and Research (IJSR) \\ ISSN (Online): 2319-7064}

Index Copernicus Value (2013): 6.14 | Impact Factor (2015): 6.391

study of both stages parameter variation and in terms of computational efficiency, recall and precision the results were analyzed. With precision of $76: 8 \%$ there achieved average recall of $91: 7 \%$ by the proposed QR code detector while at $22 \mathrm{fps}$ being capable of processing a 640 by 480 pixels video stream. For real-time applications implementation these results support which in mobile hardware assist visually impaired people and robots, through QR codes in multiple medium to the wealth of information available allowing them to have access.

\section{B. $1 D$ bar code reading on camera phones}

In this paper, using a NOKIA 7650, VGA camera phone on the bar code reading algorithms we present the research effort. From poor-quality images to extract bar code characters knowledge-based bar code segmentation and a wavelet-based barcode region location scheme is applied. For the recognition engine there are input all the characters which are segmented barcode, and the barcode character string as the final recognition result with the smallest total distance is output of the barcode based on the recognition distance. For optimizing the class reference vectors and a feature extraction matrix in order to train an efficient recognition

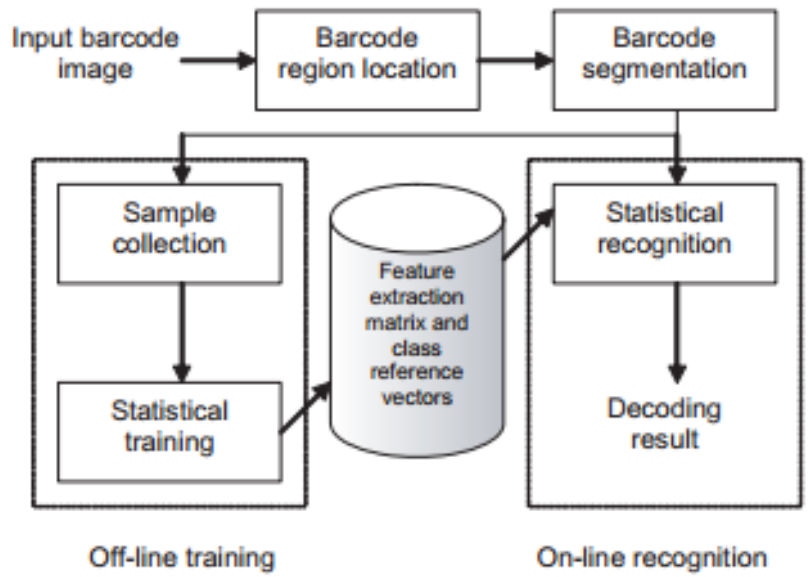

Figure 3: Diagram of the barcode decoding system

engine, there is designed the modified Generalized Learning Vector Quantization (GLVQ) method. By NOKIA 7650 from more than 1000 barcode images captured, 584 samples segmented are involved in the training process. By the same phone the testing on 292 barcode images taken, there reaches $85.62 \%$ the entire barcode set of the correct recognition rate.

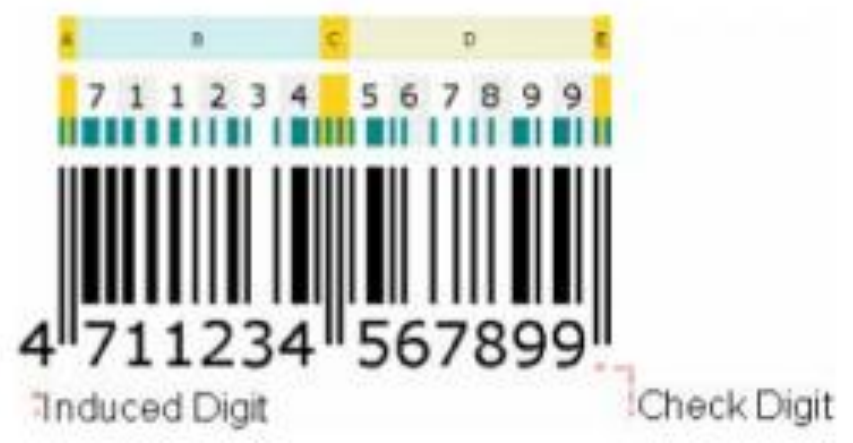

Figure 4: 1D barcode

\section{Proposed System}

Barcoding, since its evolution has emerged as a very secure and quick way to identify the data or things uniquely. But the other major use of the barcoding is to secure the data using barcodes. The concept of 2-D barcodes is of great relevance for use in wireless data transmission between handheld electronic devices. In a general setup of barcode standalone systems, any file on a cell phone, for example, can be transferred to a second cell phone through a series of images on the LCD which are then captured and decoded through the barcode scanner of the second cell phone. In the proposed system, a new approach for data modulation in high capacity 2-D barcodes is introduced, where in the High capacity $2 \mathrm{D}$ barcode of $448 * 63$ pixels is used to barcode the data and thereby transmitted through any wireless or wired medium.

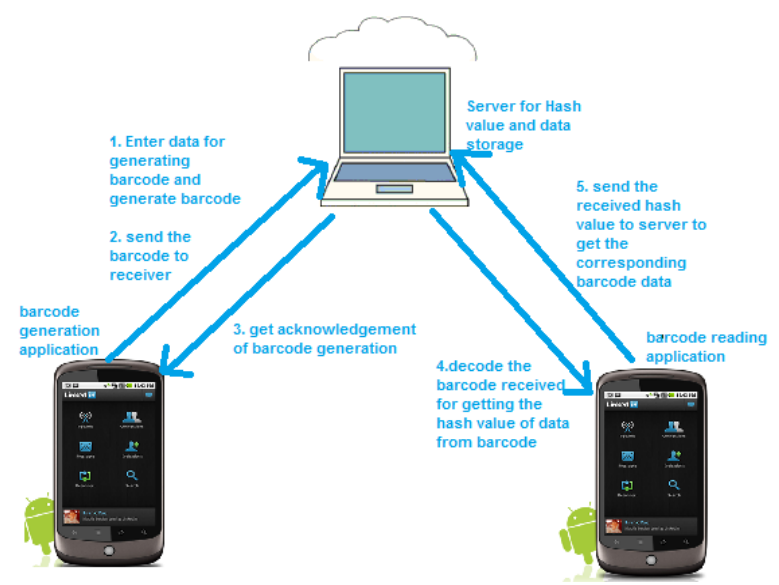

Figure 5: System Architecture

The proposed barcode has a high capacity of encoding the large amount of data by making use of MD5 secure hashing algorithm for uniquely identifying the data. The data is hashed using MD5 algorithm and thereby retrieved by cross hashing the values decoded from the barcode. The proposed barcoding and barcode modulation technique makes use of the latest technology for generating and reading the barcodes and thereby avail users to securely transmit the data. The proposed system designs a novel HC2D barcode generation technique and barcode reading technique with a secure transmission of data by making use of digital signature and data encryption before barcode encoding. The major components of the proposed systems are mentioned in the architecture diagram:

\section{HC2D barcode Generation Module}

Barcode modulation for High capacity 2 D barcode for large textual data transmission using mobile phones. Steps:

1) Initially we need to use the new technique of barcode generation and normal 2D barcodes are scanned and read by many free application in available freely.

2) So in order to secure our data, we need to design a new barcode generation and reading technique so that we no other application can read the barcode and the data is secured.

3) Also, to assure the data security, we also generate the barcode of the encrypted data, as even if the barcode is decoded, it is not in the plain text format, instead it will 


\section{International Journal of Science and Research (IJSR) \\ ISSN (Online): 2319-7064}

Index Copernicus Value (2013): 6.14 | Impact Factor (2015): 6.391

be in the Cipher text format which is to be further decrypted using the corresponding keys.

4) The aim of the system is to allow large amount of sensitive data to be transmitted to the mobile devices over internet or any other resource.

5) The sensitive data is provided two level security with encryption and the barcoding technique.

6) The new barcode specifications and details are mentioned below:

Barcode length $=448$

Barcode width $=63$

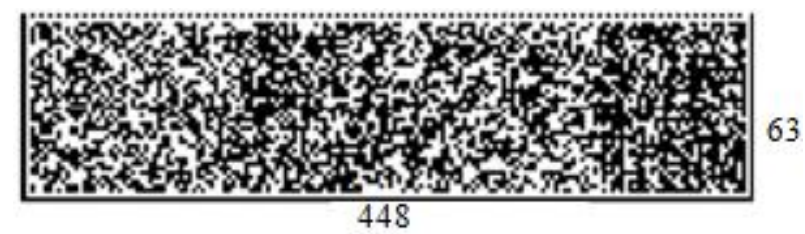

Figure 6: Sample HC2D Barcode image

We generate the 32 hexadecimal bytes of the large data using MD5 algorithm to get the unique code for the encoded data. This hash value is encoded on the barcode, and the corresponding hash value and the data entry is made in the server. While receiver has the barcode, receiver application reads the barcode and gets the hash value of the encoded data from the barcode, this hash value is then sent to the server to authenticate whether such hash value entry is done to the server. If the hash is found, the corresponding data entry made during barcode generation is sent to the receiver application.

\section{Data Transmission Module}

The major concern of the Proposed system is encoding and decoding the barcode without using any hardware, but the secondary aim of the proposed system is to transmit the generated barcode to the authorized user only and avoid unauthorized access of barcodes. The data encryption is carried out through AES algorithm as AES being highly secure for data encryption. Also, the large data reduction is done through hashing for making the validation of barcode data being encoded. Once the data is encoded, re encoding of the same data is to be avoided by checking the duplicate hash value generated before data encoding. The barcode once generated is uploaded to the server which is accessed only by the user to whom the file is to be sent.

\section{E. Set Theory representation of Operaations:}

- Sender Module:

Set $(S)=\{s 0, c o, s 1, s 2, s 3, s 4, c 1, s 5, s 6, s 7\}$

S0- send login credentials to server

S2- select file to be encode

S3- generate hash value of input file

S4- send hash value for validation

S5- encrypt the input data

S6- generate barcode

S7- upload barcode to server

- Receiver Module:

Set $(\mathrm{R})=\{\mathrm{r} 0, \mathrm{c} 0, \mathrm{r} 1, \mathrm{c} 4, \mathrm{r} 2, \mathrm{r} 3, \mathrm{c} 5, \mathrm{r} 4, \mathrm{r} 5\}$

$\mathrm{R} 0$ - send login credentials to server

R1- select barcode from uploaded barcode list

$\mathrm{R} 2$ - decode hash value from barcode
R3- send hash value for data existence

R4- decrypt the received encrypted data

R5- download decrypted data

- Server Module:

Set $(C)=\{s 0, r 0, c 0, s 4, s 7, c 1, c 2, c 3, r 1, c 4, r 3, c 5\}$

$\mathrm{C} 0$ - send login status to sender and receiver

$\mathrm{C} 1$ - send comparison status to sender

C2- check for same hash value is present or not

C3- make entry log for encoded barcode

C4- send selected barcode to receiver

C5- send encrypted data to receiver

\section{Experimental Results}

As per the proposed system implementation methodology, the efficiency of large data encoding into smaller barcode size will be a very innovative feature with barcodes being readable with only specific application having the decoding logic incorporated in it. No hardware is required to read the barcode. Especially, no hardware scanners which cost unnecessarily to the users is required. Only the android based application is sufficient for creating and decoding as well as transmitting the barcodes amongst the members being registered over the application.

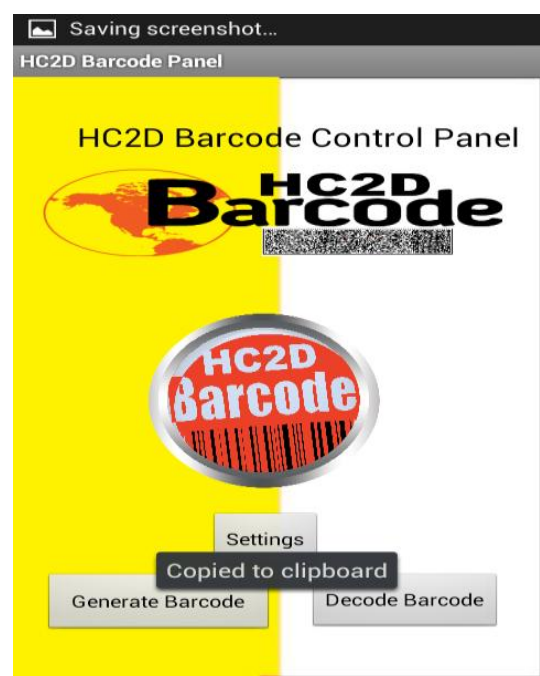

Figure 7: Home page HC2D Barcode

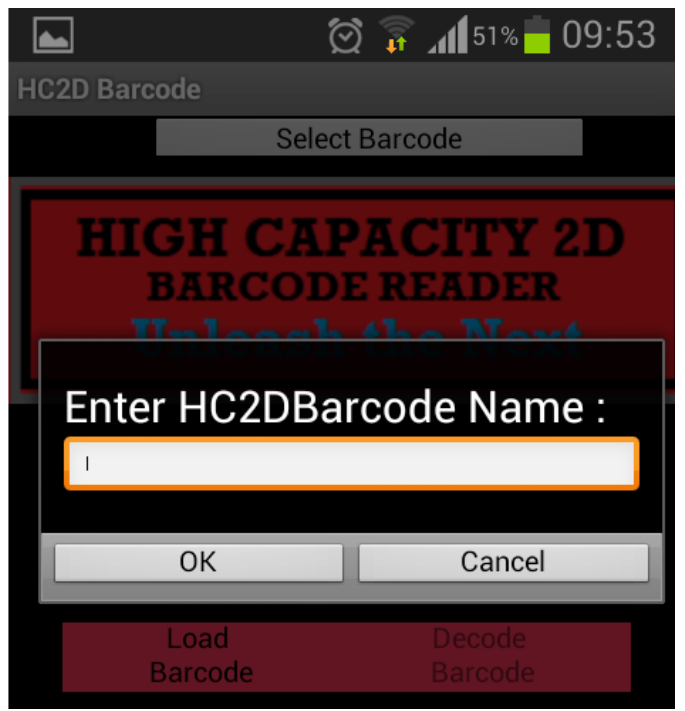

Figure 8: HC2D Barcode load screen

Volume 5 Issue 6, June 2016 www.ijsr.net 


\section{International Journal of Science and Research (IJSR) \\ ISSN (Online): 2319-7064}

Index Copernicus Value (2013): 6.14 | Impact Factor (2015): 6.391

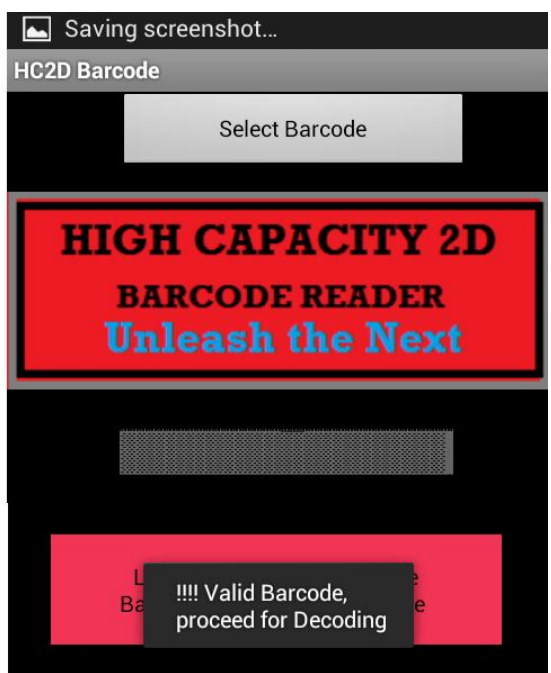

Figure 9: Barcode Loading Completed with validation
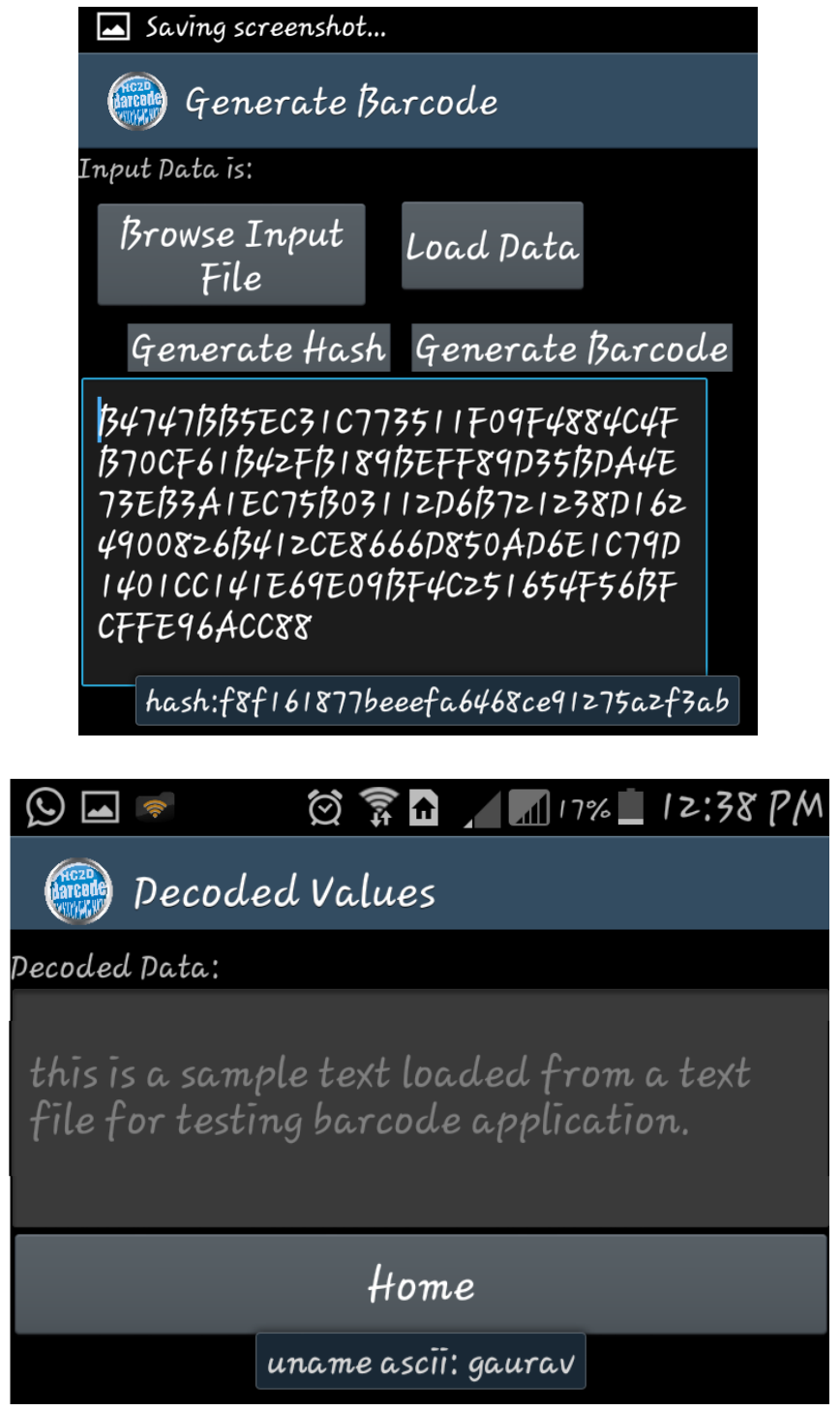

\section{Conclusion}

In this system in order to data stream modulate into visual HC2D barcodes, the combination of High capacity novel barcode generation and barcode decoding techniques carried out. As the proposed algorithm for barcode generation is quite a new technique, hardware barcode gun for decoding the barcode is not yet available and as the system is still under research, only software decoding of the generated barcode will be carried out in the proposed system. The proposed system makes the use of encrypted data modulation on to the barcodes and hence the security of the data being encoded in barcode is maintained and thus makes user convenient to transfer the large amount of data through a single barcode over the transmission medium. Thus the proposed system provides high level security to the data being modulated and transmitted with minimum network usage for larger data too.

\section{Experimental Analysis}

The implementation of the proposed system has been carried out in android with minimum android support of $2.2 \mathrm{OS}$ and PC working as a server to make the barcode scanner application interact with each other. The Experimental results show that the textual data with any input length depending upon the database size can be used as input for creating barcode. The proposed system performs the barcode creation and decoding based on MD5 hash value for determining the uniqueness of the data being encoded

\section{Acknowledgment}

I would like to take this opportunity to express my profound gratitude and deep regard to my guide Prof. Shyam Gupta for his exemplary guidance, valuable feedback and constant encouragement throughout the duration of the project. His valuable suggestions were of immense help throughout my project work. His perceptive criticism kept me working to make this project in a much better way. Working under him was an extremely knowledgeable experience for me.

\section{References}

[1] J. Memeti, F. Santos, M. Waldburger, and B. Stiller, "Data transfer using a camera and a three dimensional code," Praxis der Informa tionsverarbeitung und Kommunikation, vol. 36, no. 1, pp. 31-37, 2013.

[2] C. Pei, Z. Zhang, and S. Zhang, "Softoc: Real-time projector-wall-camera communication system," in Proc. ICCE, Jan. 2013, pp. 100-101.

[3] S. Kuzdeba, A. M. Wyglinski, and B. Hombs, "Prototype implementation of a visual communication system employing video imagery," in Proc. CCNC, 2013, pp. 184-189.

[4] M. Mondal and J. Armstrong, "Impact of linear misalignment on a spatial OFDM based pixelated system," in Proc. 18th Asia-Pacific Conf. Commun., Oct. 2012, pp. 617-622.

[5] M. Mondal and J. Armstrong, "The effect of defocus blur on a spatial OFDM optical wireless communication system," in Proc. 14th Int. Conf. Transparent Opt. Netw., Jul. 2012, pp. 1-4.

[6] M. R. H. Mondal and J. Armstrong, "Analysis of the effect of vignetting on mimo optical wireless systems using spatial OFDM,” J. Lightw. Technol., vol. 32, no. 5, pp. 922-929, Mar. 1, 2014.

[7] R. Gonzalez and R. Woods, Digital Image Processing, 3rd ed. Upper Saddle River, NJ, USA: Pearson Education, 2007.

\section{Volume 5 Issue 6, June 2016 www.ijsr.net}




\section{International Journal of Science and Research (IJSR) \\ ISSN (Online): 2319-7064}

Index Copernicus Value (2013): 6.14 | Impact Factor (2015): 6.391

[8] A. Sripad and D. Snyder, "A necessary and sufficient condition for quantization errors to be uniform and white," IEEE Trans. Acoust., Speech, Signal Process. , vol. ASSP-25, no. 5, pp. 442-448, Oct. 1977.

[9] J. Proakis and M. Salehi, Digital Communications, 5th ed. New York, NY, USA: McGraw-Hill Education, 2007.

[10] T. Cover and J. Thomas, Elements of Information Theory,2ndEd. ed. Hoboken, NJ, USA: Wiley, 2006.121-130.

Volume 5 Issue 6, June 2016 www.ijsr.net 